\title{
Interoperability of IP Multimedia Subsystems: The NetLab Approach
}

Andrés Marín-López ${ }^{1}$, Daniel Díaz-Sánchez ${ }^{1}$, Florina Almenárez-Mendoza ${ }^{1}$, Francisco Rodríguez García ${ }^{2}$, Joaquín López Rizaldos ${ }^{2}$, Alfonso Cubero Vega ${ }^{2}$, Itziar Ormaetxea ${ }^{3}$, Juan Luis Lopez ${ }^{4}$, Sergio Fernández ${ }^{4}$, Fernando Ortigosa ${ }^{4}$, Ivan Kotuliak $^{5}$, Eugen Mikoczy ${ }^{6}$, Timo Lahnalampi ${ }^{7}$, Timo Koski $^{8}$, and Sussana Avessta ${ }^{8}$

1 Universidad Carlos III de Madrid, Spain

\{amarin, dds,florina\}@it.uc3m.es

2 Telefónica Investigación y Desarrollo, Spain

$\{\mathrm{frg}$, jolo, acubero\}@tid.es

3 Software Quality Systems, Innovalia Group

iormaetxea@sqs.es

4 Ericsson Network Services, Spain

\{juanluis.lopez, sergio.fernandez, fernando.ortigosa\}@ericsson.com

5 Slovak University of Technology in Bratislava, Slovakia

ivan.kotuliak@stuba.sk

6 Slovak Telekom, Slovakia

eugen.mikoczy@t-com.sk

7 Digital Media Service Innovations, Finland

timo.lahnalampi@dimes.fi

8 University of Turku, Finland

\{timo.koski, annys\}@utu.fi

\begin{abstract}
Standardization bodies have spent lots of efforts and have extensively defined IP Multimedia Subsystem interfaces. While Telcos have started its deployment, IMS-based applications boosting user needs are still to come. Such applications must not suffer from interoperability issues caused by different vendors and different administrative domains. NetLab project aims at exploring such interoperability issues by interconnecting together testbeds at three different countries. Use cases will be defined to find out about interoperability, and also to search for a user appealing application in collaboration with the LivingLabs community. This paper introduces the project vision on the architectural, security, QoS, and interoperability issues, together with new services illustrating the interconnection of testbeds.
\end{abstract}

Keywords: IMS interoperability, authentication and QoS in IMS, Living Labs.

\section{Introduction}

Next Generation Networks (NGN) are considered as a major step in the telecommunication world evolution. The novelty consists in the transition of the core

J. Wozniak et al. (Eds.): WMNC 2009, IFIP AICT 308, pp. 20-31 2009.

(C) IFIP International Federation for Information Processing 2009 
network from the PSTN (Public Switched Telephone Network) to the all-IP with QoS management. Advantages for the operators go from simplifying the core network using less complex equipment to the cost saving (both CAPEX and OPEX). Telecom operators have rapidly understood these advantages and started to replace PSTN by NGN.

The deployed equipment has been conformed to the soft-switch architecture from the beginning. The soft-switch represents a monolithic central block, which handles whole signalization and controls gateways. Such approach is rather inflexible. During the time, 3GPP has started standardization of the NGN implementation in the mobile networks as IP Multimedia Subsystem (IMS) [1]. It appeared in release 5 and now it has become a widely accepted standard and architecture for the NGN implementation (also for fixed networks). The main enhancement of the IMS compared to the soft-switch is the distribution of the functions into different nodes and the definition of interface points. Thanks to this, Telcos can use different functions from respective vendors and the crucial point is that applications have become completely open to the 3rd party developers.

The IMS is already well standardized. However, the applications and their behavior in the various interconnect scenarios are still under heavy understudy. The aim of the NetLab project is to develop a platform of interconnected test beds involving three different countries together with different Living Labs. Living Lab is an experimentation environment in which technology is given shape in real life contexts and in which (end-) users are considered 'co-producers'. Living Lab involve human-centric development of new ICT-based services and products. Panlab project has developed a federation framework of interconnects testbeds belonging to regional clusters, hence partitioning the management of the resources to different levels. The main design principle in Panlab federation is the dedication of resources under the common federation governance. NetLab platform should be designed to tackle interoperability, scalability, complexity and mobility aspects holding security and QoS requirements. Such an approach allows refining Panlab/Living Lab concepts with respect to different issues (organization, business models, legal aspects, security, IP protection) and to develop 2 IMS and DVB-H related use cases involving Panlab and Living Labs scenarios. The NetLab project is complementary to both Panlab and LivingLab, where Panlab testbeds have a technological focus, and Living Labs a user centric focus.

The organization of the article is as follows: section 2 covers the architectural issues including security and QoS aspects, and the overview of the platform interconnection possibilities, section 3 describes Netlab proposal for new services and use cases, and section 4 presents the related works. Finally, we end with some conclusions.

\section{Architectural Issues}

The development of IMS services is currently ongoing, but the services of different vendors are still lacking on the interoperability. NetLab's interconnected testbed architecture will support IMS service and application developers by 
providing different testing environments under one agreement and system. The architecture shall support service and technology developers of both the owner parties of testbeds and external parties in need of testing environment. There are two main issues in approaching the architecture goal.

First, the interoperable parts of different testbeds need to be defined in order to be able to run end-to-end services across. This includes a study of service scenarios and use cases. Based on them, a profile of interoperable services and applications is defined. This activity will lead to a service catalog of possible applications and their availability in different testbeds, and definition of methods how to access them.

Second, both organizational and technical access methods to testbeds need to be defined. This includes interfaces for both interconnection between users of testbed organizations and external users. On the organizational level, usage rights are defined in an interdomain agreement between parties. The interdomain agreement is supported on the technical level by definition of methods for authorization, authentication and accounting (AAA). Based on the method selection, common protocols for both AAA and service access will be defined, from the network layer and up. Other technical issue in definition of the architecture is the requirement of maintaining a sufficient QoS (Quality of Service) level.

\subsection{Security Aspects}

TISPAN (Telecommunications and Internet converged Services and Protocols for Advanced Networking) and 3GPP (3rd Generation Partnership Project), defined different Transport Control Layers for IMS; this has not contributed to a full fixed-mobile convergence.

In the fixed domain TISPAN has adhered to traditional authentication solutions, where dial-up protocols (mainly Point-to-Point Protocol, PPP) are used as authentication methods in the physical line where user is connected and identified. 3GPP also maintains, in mobile networks, Global System for Mobile communications (GSM) mechanisms, improving slightly security issues. These different approaches lead to having two disparate authentication models based on different protocols, different identities, different infrastructure and different user provision processes.

NetLab project main results will be in the area of interoperability and interconnection of testbeds. Besides, we will explore the compatibility issues of different terminals and IMS clients with different IMS cores. Some of our first results have lead us to explore in the security issues and requirements of the system. Once IMS deployments and IMS services become as widespread as 3G services today, we will be prepared to give answer to security issues we will explore in NetLab such as: achieving fast user authorization with IMS application services, specially for nomadic and roaming users, protecting users from rogue visiting networks, security configurations guaranteeing users and Telcos the quality of authentication, and integration of legacy conditional access systems protecting multimedia contents in IMS enabled IPTv services. 
NetLab project proposes a novel AAA convergent architecture. Using the same authentication algorithms is needed in order to achieve a common platform. Since authentication algorithms for fixed networks have not evolved in the last decades and drag out some drawbacks (for example, user identity is attached to the physical line, not allowing nomadism and adding complexity to provisioning systems), mobile networks authentication mechanisms are proposed. Two main methods are used nowadays in mobile networks: Subscriber Identity Module (SIM [3]) and Authentication and Key Agreement (AKA [4]), both of them based on Universal Integrated Circuit Card (UICC), using . Nevertheless, due to the different physical access mediums used by fixed and mobile networks, we will experiment with a different authentication transport protocol for fixed networks: 802.1X [2]. Fig. 1] shows the AAA architecture proposed.

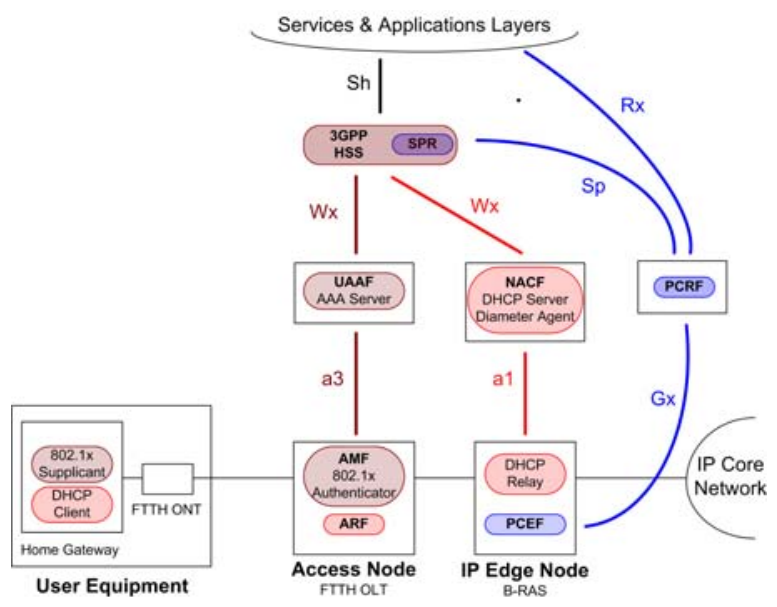

Fig. 1. Convergent AAA Architecture

\subsection{QoS Aspects}

Most ISPs pursue providing both user QoS requirements and QoS admission control. To provide user applications QoS requirements implies granting end-toend resources in order to provide a packet delivery that meets the application requirements for every traffic flow, such as bandwidth, delay, packet loss, jitter, etc. Providing QoS admission control implies taking into account when the network is not going to be able to grant a given user application traffic flow QoS requirements and permits the operator executing the corresponding action according to a defined policy (for example, do not permit to establish the given traffic flow or inform to the end-user that the network is unable to assign the required QoS resources and the application quality could be degraded).

Standardization bodies, such as TISPAN in fixed networks and 3GPP in mobile ones, have done an attempt to achieve these targets. Since effective end-toend QoS mechanisms (e.g. RSVP) do not provide operator-managed admission 


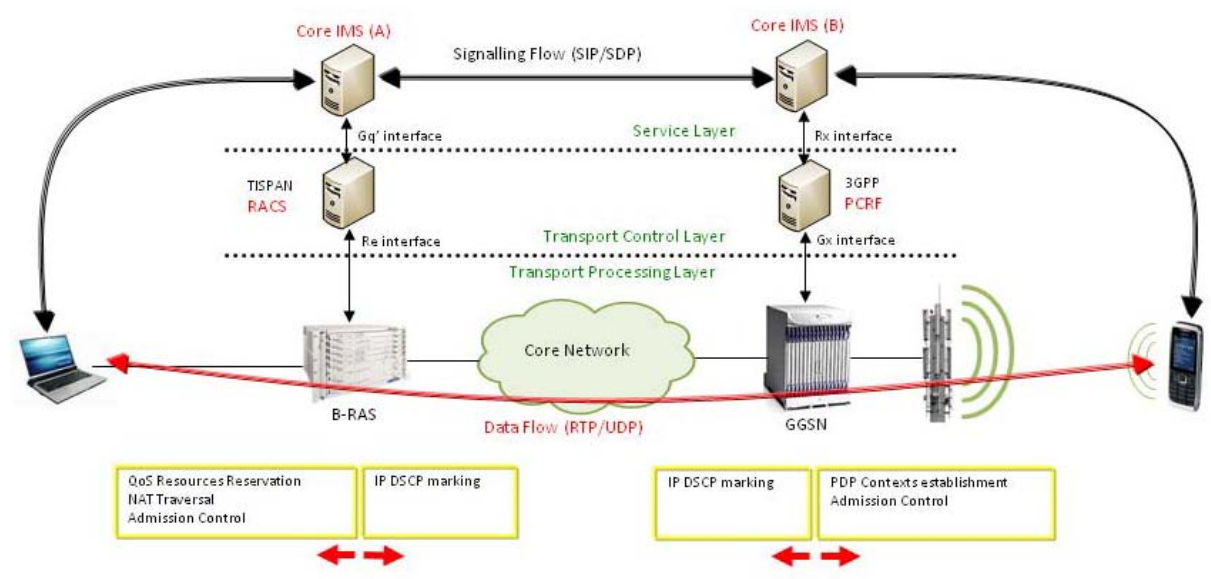

Fig. 2. QoS High-Level Description

control techniques, both TISPAN and 3GPP have considered that the access network is the bottleneck (assuming that the core network is over dimensioned) and have focused all his efforts on implementing both QoS resources reservation and QoS admission control in the access network. In the last years many efforts have been devoted in order to design a convergent QoS Architecture taking as a starting point both TISPAN and 3GPP proposals. Nevertheless, this goal has not been achieved yet, and the last attempt carried by 3GPP (PCRF definition) is still in progress. NetLab project will extend the current QoS platforms in order to achieve a convergent architecture, getting a common transport layer for both mobile and fixed networks enabling a network-agnostic service layer (see Fig. 2).

Regarding that new broadband access technologies, such as FTTH and LTE, will supply a dramatically increase in the user available bandwidth, standardization bodies' premises are not longer valid. The bottleneck could be located in different locations, such as inside the user home network or even in the core network.

Traffic flows IP DSCP marking is proposed in NetLab project, so that realtime traffic flows (used in conversational services) will be prioritized over some other background flows (such as web navigation) in the whole network (home user network, access network and core network).

Bottlenecks could also appear in layer-2 devices, such as Wi-Fi access points or PLC devices, which do not implement layer-3 features and are not able to prioritize high-priority traffic since will not detect DSCP flags. To grant user QoS requirements in these devices, layer-2 flags will be also marked, classifying traffic flows according to standards such as WMM and Ethernet 802.1p. NetLab project defines 4 QoS traffic classes (Voice, Video, Best-Effort and Background) and every traffic flow will be categorized in one of these classes, providing QoS resource reservation and admission control for IMS services in the access network and prioritizing high-priority traffic flows in the whole network in order to provide end-to-end QoS in case the bottleneck is located in other network segment. 


\subsection{Platform Interconnection}

Interconnection between testbeds provides end user access to all available services which are provided also by partners testbeds over home network (own operator).

\section{Co-operation with European Testbeds: net lab}

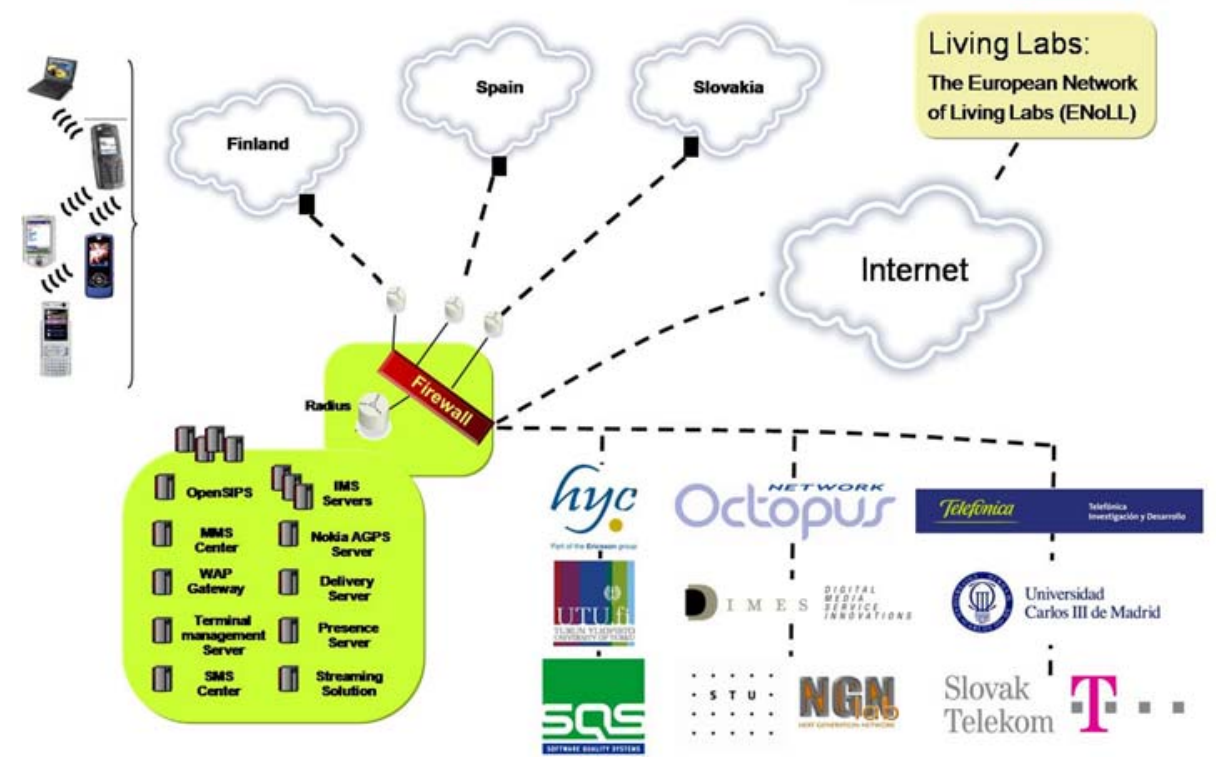

Fig. 3. Platform High-Level Interconnection

There are several possible way of IMS or NGN interconnection in the standards (e.g. those from ETSI TISPAN, 3GPP). Additional to this there are some specifications for IMS interconnect which should be adapted and considered. 3rd Generation Partnership Project 3GPP: Interconnection IPv4 vs. IPv6 (dual models) also deals with NAT and IP address incompatibility [6]; interconnection to pure SIP domain[7]; ETSI Telecommunications and Internet converged Services and Protocols for Advanced Networking (TISPAN): new work items for NGN interconnection (NGN architecture R2 [9], IMS based IPTV 8 ), and GSM Association: IMS Roaming and interworking [10[11].

A interconnection scenario fully based on standards requires some additional components: located at the transport layer, an Interconnection Border Gateway Function (IBGF) is needed; at the signaling layer, an Interconnection Border Control Function (IBCF) with co-located Interworking Function (IWF) are used. The main functions in the transport layer are traffic control, Topology Hiding (THIG) and Bearer Control (BC). The IBCF uses THIG for signaling information and controls the IBGF through the Service based Policy Decision Function 
(SPDF). This SPDF performs a coordination function acting as Policy Decision Point for Service-based Policy control (see also [5]). It makes policy decisions depending on service policy rules defined by the network operator. Furthermore, the underlying network technology will be hidden from applications.

The authorization of application functions requesting transport control service is based on a process, which involves the check of these requests against the given policy. The IBCF and IBGF control each session including signaling data and media data, as all traffic will be routed through these two components. Acting as entry point, both entities are configured to forward the signaling data to the I-CSCF for new sessions and to the P/S-CSCF for existing sessions. Media data will be routed to an Access Network Gateway Function (ANGF) or Media Gateway Function (MGF) for established connections. Outgoing traffic should be forwarded to an IBCF connected to the target domain for signaling information and media data to the corresponding IBGF.

Steps from plain to standardized Interconnection
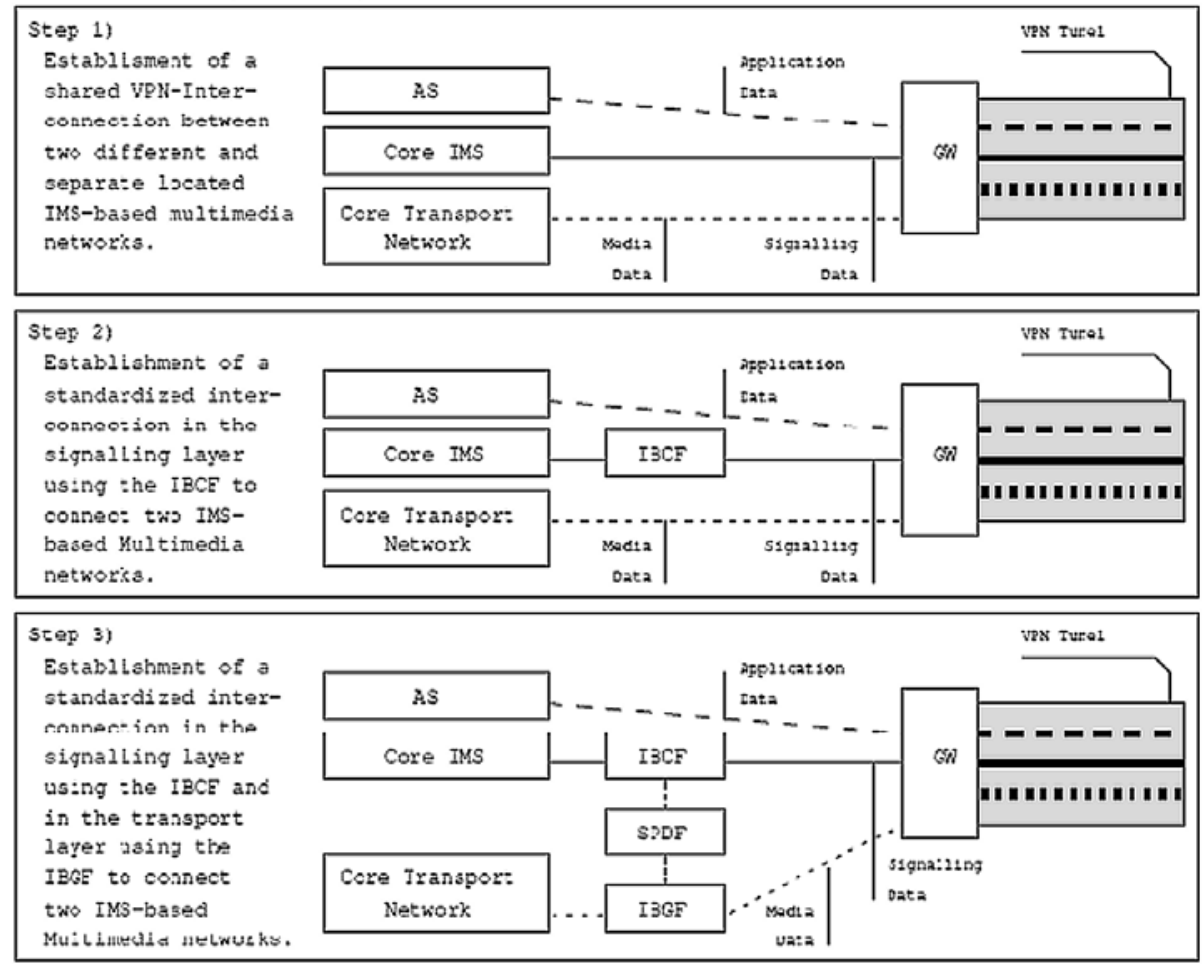

\footnotetext{
Legend: AS - Application Server

IRCE - Interconnection Border ControL Eunction

SYDE - Service-based yolicy vecision Foution

IBGE - Interconnection Bcrde $y$ Gateway Eurction
}

Fig. 4. Possible interconnection of IMS testbeds, GEANT connections not shown 
As first steps the interconnection of partners testbeds will depend on available components, but most probably an interconnection without all the entities as described in standards can be established first and later extended. This is seen as first and simplest approach to show, so that servers of interconnected testbeds can communicate in spite of the difference of environments and that the standard compliant interconnection has a solid base. All required steps for a grateful changing of the actual running configuration to a standardized interconnection in the future are shown in Fig. 4. Take into account that the VPN interconnection will be later substituted by MPLS tunnels over the GEANT network.

The proposed set-up allows sharing of Application Servers (AS), which are located in different domains. Using the presence service, two different interfaces are shared and forwarded between the testbeds. ISC/Ma is the first interface. It will be used to exchange information between the Serving (S)-CSCF and the Presence-AS. The latter interconnection is established on the Ut interface, used by a User Agent (UA).

\section{Services and Use Cases}

Currently operators are able to run IMS and DVB-H services on their own platforms, but when time comes to interconnect infrastructures, problems have to be solved to deal with service interoperation, orchestration, roaming and end-to-end QoS (Quality Of Service). The objective of NETLAB is to have services working on top of the multi-platform and multi-operator interconnected environment; the result is to enable real life tests and to provide field materials to conduce theoretical work on service modeling and testing.

To define the services, focus has been put on both the application servers side and the terminal clients side. The application servers implement the service logic, and in a multi-platform and multi-operator environment, interoperation, orchestration or roaming problems have to be solved. The terminal clients negotiate end-to-end QoS, and serious interoperability problems appear when interconnecting two access networks with different technologies (most notably, mobile and fixed), because the QoS signalling mechanisms are not totally compatible.

To deliver adapted services to the real-life experiments and to address interesting problems for theoretical work, suitable use cases have been defined. The focus was put on IMS services that have to run on top of a multi-platform and multi-operator environment. Basic IMS services such as "VoIP", "Presence" or "Instant Messaging" are required, but other services such as "Push to Talk" or "IP TV" were addressed.

Once services have been deployed on interconnected platforms, they have to be tested and benchmarked at the operator level. The objective is to validate that a given service interoperates properly, that it works in roaming context and that end-to-end security and QoS are achieved. Before services are delivered for real-life testing, the use case services must be adapted to the targeted platform environments, the terminal clients should be adapted to fix QoS interoperability problems; and the services are deployed on the IMS platforms; the services are recursively tested, at the operator level, to fix interoperability problems. 
NetLab project proposes different services to improve the interoperability among different testbeds (under different administrative domains, using different implementations, different vendors, equipment, etc.). Some of the proposed services on the interconnected testbeds are the following:

\section{Integrated rich communication with IPTV}

The rich communication services provide basic set of services expected by operators from IMS based NGN in our scenarios will be evaluated potential use cases where show advantages for integration of voice/video communication combined with messaging, presence, buddy list cross domains and beyond usual IMS roaming scenarios. Main difference will be achieved by accessibility of services also outside home network where applications can be provided and combines by multiple platforms application servers and enable use e.g. presence cross domains. We would like to show also possible integration of rich communication with content services and IPTV.

\section{IPTV service remote control}

This service will allow remote controlling IPTV services (e.g. parental control). The kids will be able to get the permission to watch some content even if the parents are not at home. The aim is to show how the traditional IPTV service can be integrated with other networks and services (e.g. SMS) through the IMS core network to improve user experience and conformity in usage. At the same time, this service also represents a scenario where the interconnection of different domains is required, especially when user want control his home services from external domain.

\section{Advanced PVR}

The main aim of this scenario is to allow the interconnection of two different accesses networks, in this case we will interconnect the mobile phone network together with the IPTV network to allow a new innovative and interesting service which consists on being able to schedule and access a recordings remotely.

\section{QoS over heterogeneous networks}

The target of this scenario is to demonstrate how end-to-end QoS resources could be granted in an IMS-based service where traffic flows across heterogeneous networks. In this scenario, IMS service layer invokes the corresponding QoS resources reservation procedures in different interconnected domains in order to grant end-to-end QoS.

\section{Unified authentication}

The main goal to achieve in this scenario is to show how a unified authentication and authorization mechanism can be used for both mobile and fixed accesses. In this way, a unique identity will identify the end user and all services will be provided independently whether the user is accessing through a fixed or mobile network and from home or visited network.

\section{Extended DLNA}

DLNA standards have been very successful and nowadays most consumer electronic devices are incorporating the functionalities proposed by DLNA, especially 
those regarding to content and media sharing. Nevertheless, DLNA standards are reduced to local area networks and features to share contents through wide area networks are not included. The main goal of this innovative service is to extend DLNA scope to reach contents share in remote devices. IMS capabilities will be used to publish DLNA media sharing features and to authorize and control remote access to local contents. IMS signaling stage will be extended to request the control layer to apply the required network configuration which allows DLNA media sharing through WAN.

\section{Related Works}

ENoLL (www.openlvinglabs.eu) was launched in November 2006 in Helsinki with 19 members at the first phase, the crew grew next year into 50 during Portuguese presidency and finally French presidency in November 2008 embraced altogether 129 Living Labs across the whole world as a result of the third wave of applications and their evaluation.

Living Lab is the concretisation of human-centric involvement for development of new ICT-based services and products. This is done by bringing different stakeholders together in a co-creative way. This is normally a very local set-up; an area or a region is establishing a living lab to foster its development and to improve the life of its citizens.

Since Living Lab is about people together for better tomorrow, scaling up the concept and jointly benefiting from the power of co-creation is fundamental for the philosophy to be put in action. Hence the Network of Living Labs. Despite the idea of living labs born in US, the network is a European concept, which now in the third wave grows global and beyond European borders. This again is only coherent with the ideology of joining relevant communities fostering co-creation.

Despite the need from the market, to the date Living Labs have not been connected to run comparable tests across multiple Living Labs. NetLab will pioneer in this task and utilize the ENoLLnetwork.

Interconnecting testbeds for a richer research and development environment is currently a global initiative, but especially advanced in Europe. National clusters have emerged and Framework Programme has targeted resources for creating large-scale research infrastructure and federating testbeds. Fig. 5 compares different related initiatives.

OneLab2 builds on PlanetLab (US) federation principle, runs independent management entity PlanetLab Europe, and gradually extends and broadens the offerings of the global networking research infrastructure by federating more nodes, introducing novel tools, but also new heterogeneous resources via vertical interconnection and new incentive models for sharing and running the testing facilities.

PII, also known as Panlab II, implements the federation framework developed previously and interconnects testbeds belonging to regional clusters, hence partitioning the management of the resources to different levels. The main design principle in PII federation is the dedication of resources under the common federation governance. PII framework addresses heterogeneous resources offered to 


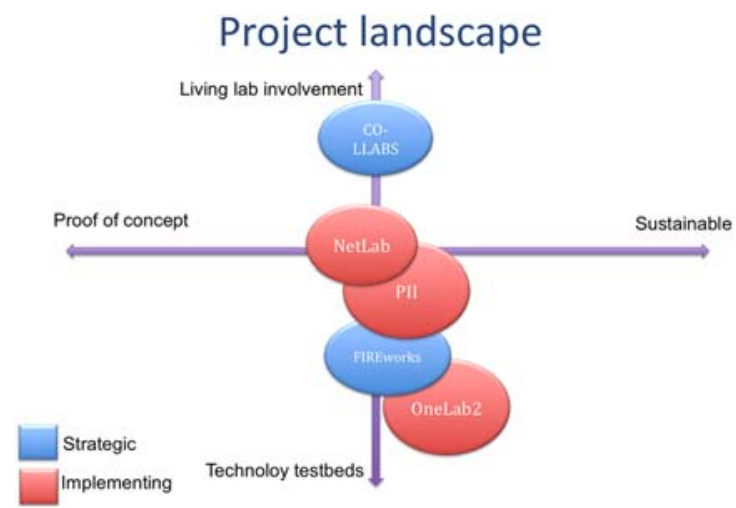

Fig. 5. Related initiatives

customers meeting also more business specific needs and criteria, such as SLAs. The proof of concept federation is implemented on IMS testbeds at three sites in Europe. The concept of User-Driven Innovation is included as PII offerings and the whole implementation process is analyzed techno-socio-economically. NetLab project approach is more pragmatic by connecting and piloting IMS test beds interconnectivity and testing new concepts related to Living Lab and Panlab concepts.

CO-LLABS and FIREworks are coordinating projects enabling more effective dissemination and resource allocation within testbed and living lab community.

\section{Conclusions}

NetLab is a cross domain platform of interconnected testbeds from three EU countries (Finland, Spain, Slovakia), together with interconnection to different European tested environments (projects like Panlab, Living Labs or overall framework FIREworks). NetLab is designed to tackle converged services over IMS based testbeds with focus on interoperability, scalability, complexity and mobility aspects as well as security and QoS requirements, coupled with validation in large scale testing environments. Use cases have been defined within the project to find out the interoperability issues from a more practical perspective.

Acknowledgments. This work has been partially supported by CELTIC NetLab Project ID CP5-018 under Spanish Science and Innovation Ministry Grant No:TSI-020400-2008-108.

\section{References}

1. 3GPP recomendation 23.228: IP Multimedia Subsystem (IMS), stage 2 (2009)

2. IEEE Standard for Local and metropolitan area networks; Port-based Network Access Control, IEEE Standard 802.1X (December 2004) 
3. Haverinen, H. (Nokia), Salowey, J. (Cisco Systems): RFC 4186; Extensible Authentication Protocol Method for Global System for Mobile Communications (GSM) Subscriber Identity Modules (EAP-SIM) (January 2006)

4. Arkko, J. (Ericsson), Haverinen, H. (Nokia): RFC 4187; Extensible Authentication Protocol Method for 3rd Generation Authentication and Key Agreement (EAPAKA) (January 2006)

5. Aboba, B. (Microsoft), Blunk, L. (Merit Network, Inc.), Vollbrecht, J. (Vollbrecht Consulting LLC), Carlson, J. (Sun), Levkowetz, H. (ipUnplugged): RFC 3748; Extensible Authentication Protocol (EAP) (June 2004)

6. 3GPP TSG Services and System Aspects: Interworking aspects and migration scenarios for IPv4-based IP Multimedia Subsystem (IMS) implementations (Release 8) TR 23.981 V8.0.0 (December 2008)

7. 3GPP TSG Core Network and Terminals: Interworking between the IM CN subsystem and IP networks (Release 8) TS 29.162 v8.1.0 (December 2008)

8. ETSI, TISPAN IPTV Architecture; IPTV functions supported by the IMS subsytem RTS 182027 v2.2.0 (November 2008)

9. ETSI, TISPAN NGN Functional Architecture. Section 7 "NGN Interconnection". ETSI ES 282001 v2.0.0 (March 2008)

10. GSM Association: IMS Roaming \& Interworking Guidelines v3.6 (November 2006)

11. GSM Association: A guide to GSMA's SIP Trials focusing on practical interworking of IMS over the evolved GRX (February 2007)

12. Lopez, J., Rodriguez, F., Fandino, A., Palacios, J.M., Garcia, A., Gonzalez, F.: Smart Card based Authentication Mechanism for FTTH Access Networks. In: The 16th IEEE Workshop on Local and Metropolitan Area Networks, LANMAN 2008, Cluj-Napoca (September 2008)

13. Lopez, J., Fandino, A., Garcia, A., Palacios, J.M., Rodriguez, F., Gonzalez, F.: Proposal for a Convergent Authentication and Authorisation Infrastructure in NGN. In: 47th FITCE Congress, London (September 2008) 